\title{
AEROSOL TRANSPORT IN A MODEL OF HUMAN LUNGS
}

\author{
Frantisek LIZAL ${ }^{1}$, Jan JEDELSKY, Josef LIPPAY ${ }^{2}$, Tereza HALASOVA, Filip MRAVEC, \\ Miroslav JICHA
}

\begin{abstract}
Complex flow structures emerging in human lungs significantly affect deposition of inhaled particles. Therefore flow measurements alongside with deposition measurements have to be performed on the same model geometry. Amount of deposited aerosol particles in different regions of lungs could be measured by several methods. Gamma detection of radioactive tagged particles is subjected to strict regulations and is expensive; gravimetry and fluorometry are thus preferred in current studies.

Novel methodology for fluorometric measurement of aerosol transport in models of human lungs was developed. Some previously published experiments were performed using condensation of di(2-ethylhexyl) sebacate (DEHS) vapours on fluorescein nuclei. Their authors assumed that fluorescein is necessary for generation of fluorescent particles and they neglected possible fluorescence of DEHS itself. Our analysis of DEHS characteristics indicated that DEHS fluorescence could be detected apart in sufficient rate to evaluate aerosol deposition. Consequently experiments in standard setup of condensation monodisperse aerosol generator with sodium chloride nuclei instead of fluorescein were performed. Results demonstrating applicability and limitations of DEHS particles for fluorometric deposition measurements are presented and discussed.
\end{abstract}

\section{INTRODUCTION}

Treatment of different lung diseases requires targeted delivery of medication to specific regions within human lungs [1]. Cancer develops usually in upper respiratory airways [2, 3], whereas asthma, chronic obstructive pulmonary disease (COPD) or cystic fibrosis (CF) requires targeting of aerosolized medication to lower respiratory airways [4-6]. There is also a great potential for delivery of systemic medication directly to the blood circulation across alveoli [7].

Fate of inhaled particles depends on many factors such as shape and density of particles, airway dimensions and their constrictions caused by lung diseases, chemical properties of particles and mucus, method of inhalation and others. Many different methods are available

${ }^{1}$ Frantisek Lizal, Jan Jedelsky, Miroslav Jicha, Brno University of Technology, Faculty of Mechanical Engineering, Technicka 2, 61669 Brno, Czech Republic, ylizal00@stud.fme.vutbr.cz

2 Josef Lippay, Tereza Halasova, Filip Mravec, Brno University of Technology, Faculty of Chemistry, Purkynova 118, 61200 Brno, Czech Republic, xchalasova@fch.vutbr.cz 
to study absorption and deposition of particles [8]. Our work was focused on investigation of aerosol deposition in human lungs. Experiments were performed on the same geometry as phase Doppler anemometry measurements of particles velocity [9] and numerical simulations of flow and deposition [10].

Deposition of aerosol could be measured by radiolabelling of particles with consequent measurement of radioactivity in particular regions of lungs. This method can be used for both, in vivo and in vitro experiments. However using radioactive material is subjected to strict regulations and also financial expenses are higher compared to other methods. Therefore we decided to use fluorometry to measure regional deposition in a model of human lungs.

\section{Methodology}

Fluorometry is based on measurement of fluorescence intensity in samples created by rinsing of particular segments of the model containing deposited luminiscent aerosol. Formerly published experiments were performed using di(2-ethylhexyl) sebacate (DEHS) particles with fluorescein sodium salt (uranin) cores as fluorescent tracer [11, 12]. Our preliminary experiments indicated that there is a possibility to detect the fluorescence of DEHS itself and therefore standard setup of aerosol generator with sodium chloride cores could be used, which is effortless and cleaner. To confirm aplicability of DEHS fluorescence for evaluation of aerosol deposition, we have performed a set of tests which are presented herein.

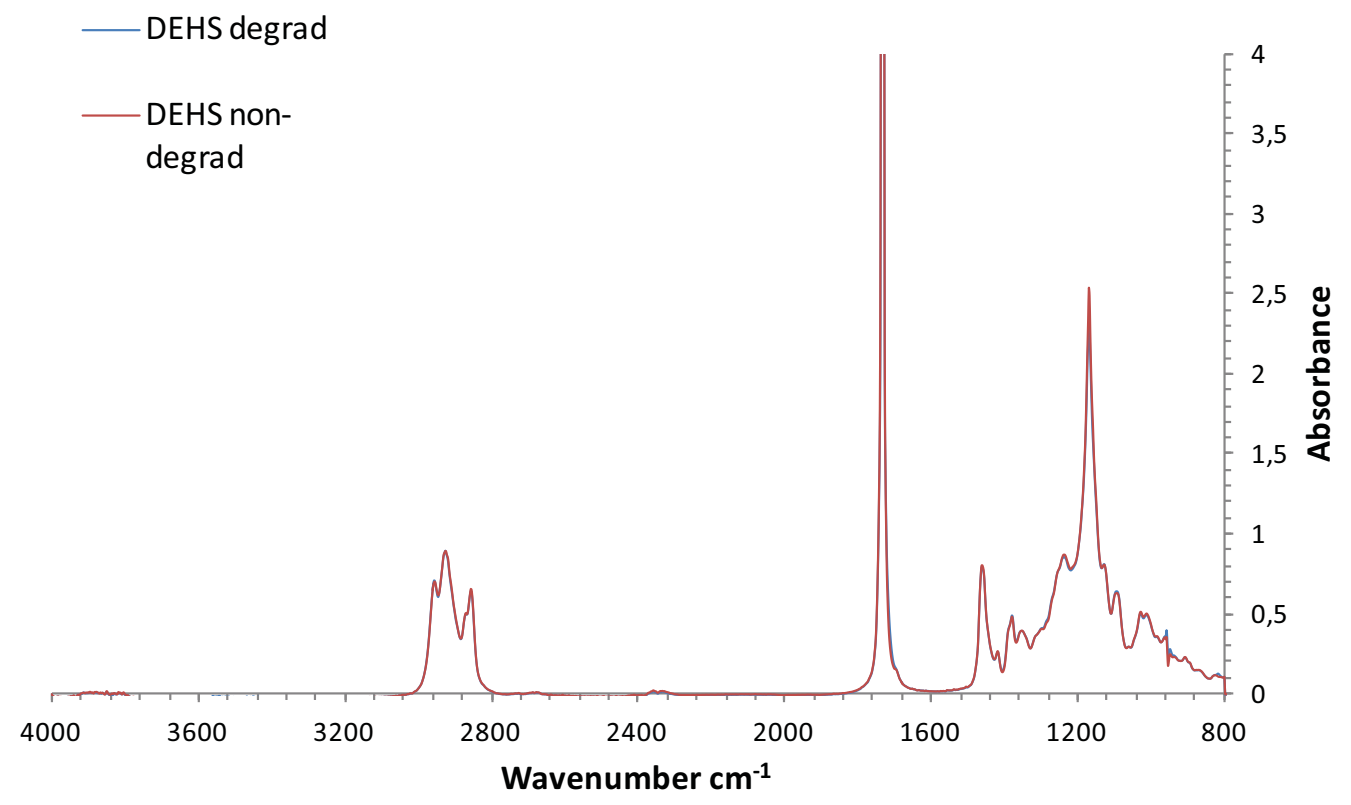

Figure 1: Infrared spectrum of distilled and degraded DEHS [13]

The first question that arises is what causes the fluorescence of DEHS. Fluorescence is an emission of light caused by relaxation of an orbital electron to a ground state after excitation 
to a higher quantum state by light at smaller wavelength. Regrettably DEHS is not a typical fluorophore and there is no obvious reason for its fluorescence, neither a note in the literature. Therefore we had to exclude a possibility of occurence of fluorescent impurities.

DEHS was distilled in low pressure atmosphere to remove possible impurities and put to test on infrared spectroscopy at Nicolet Impact 400 with ATR crystal. The infrared spectrum of a sample is recorded by passing a beam of infrared light through the sample. When the frequency of the IR light is the same as the vibrational frequency of a bond, absorption occurs. Examination of the transmitted light reveals how much energy was absorbed at each wavelength. From this, a transmittance or absorbance spectrum can be produced, showing at which IR wavelengths the sample absorbs. Analysis of these absorption characteristics reveals details about the molecular structure of the sample [14]. IR spectrometry is an instrument for highly sensitive detection of different chemical elements and is often used in forensic science. We can expect that IR spectrometry will easily detect possible impurities in DEHS. However Figure 1 illustrates that IR spectrum of distilled and original DEHS does not differ; therefore we can assume that there are no significant organic impurities present in the samples.

It follows from our experience that DEHS changes its colour from almost transparent with a tinge of yellow to yellow color during experiments due to heating in aerosol generator. There is obviously some kind of degradation which should be recorded by fluorometry or possibly by infrared spectroscopy. Regrettably infrared spectroscopy gave the same spectra for degraded and non-degraded DEHS, therefore these two DEHS types can not be distinguished by this method. 3D fluorescent spectrometry was used for verification of the supposition that there are two types of DEHS: non-degraded and degraded. Excitation scans were performed for wavelengths $250 \mathrm{~nm}$ to $500 \mathrm{~nm}$ with $5 \mathrm{~nm}$ step and emission scans were performed for wavelengths $250 \mathrm{~nm} 600 \mathrm{~nm}$ with $10 \mathrm{~nm}$ step. Resulting scans are presented in Figure 2 and Figure 3.

The methodology of aerosol deposition measurement requires usage of suitable solvent to dissolve DEHS deposited on each segment. Seven solvents were tested: isopropanol, ethanol, methanol, dimethyl sulfoxide (DMSO), cyclohexane, xylene and chloroform.

Fluorescence characteristics of DEHS in different solvents was investigated by fluorescence spectroscopy on AMINCO Bowman Series 2 spectrometer. Samples were created by dissolving of $0.5 \mathrm{ml}$ of DEHS in $4.5 \mathrm{ml}$ of solvent and mixed for 24 hours. The samples were 3D scanned afterwards.

The best yield was achieved with isopropanol, the worst in xylene (see Figure $\mathbf{4}$ and Figure 5). Isopropanol therefore appears as the best solvent for DEHS.

As these tests confirmed detectability of DEHS-isopropanol solution fluorescence, we decided to perform deposition experiments with DEHS aerosol in standard setup of aerosol generator with sodium chloride nuclei. 


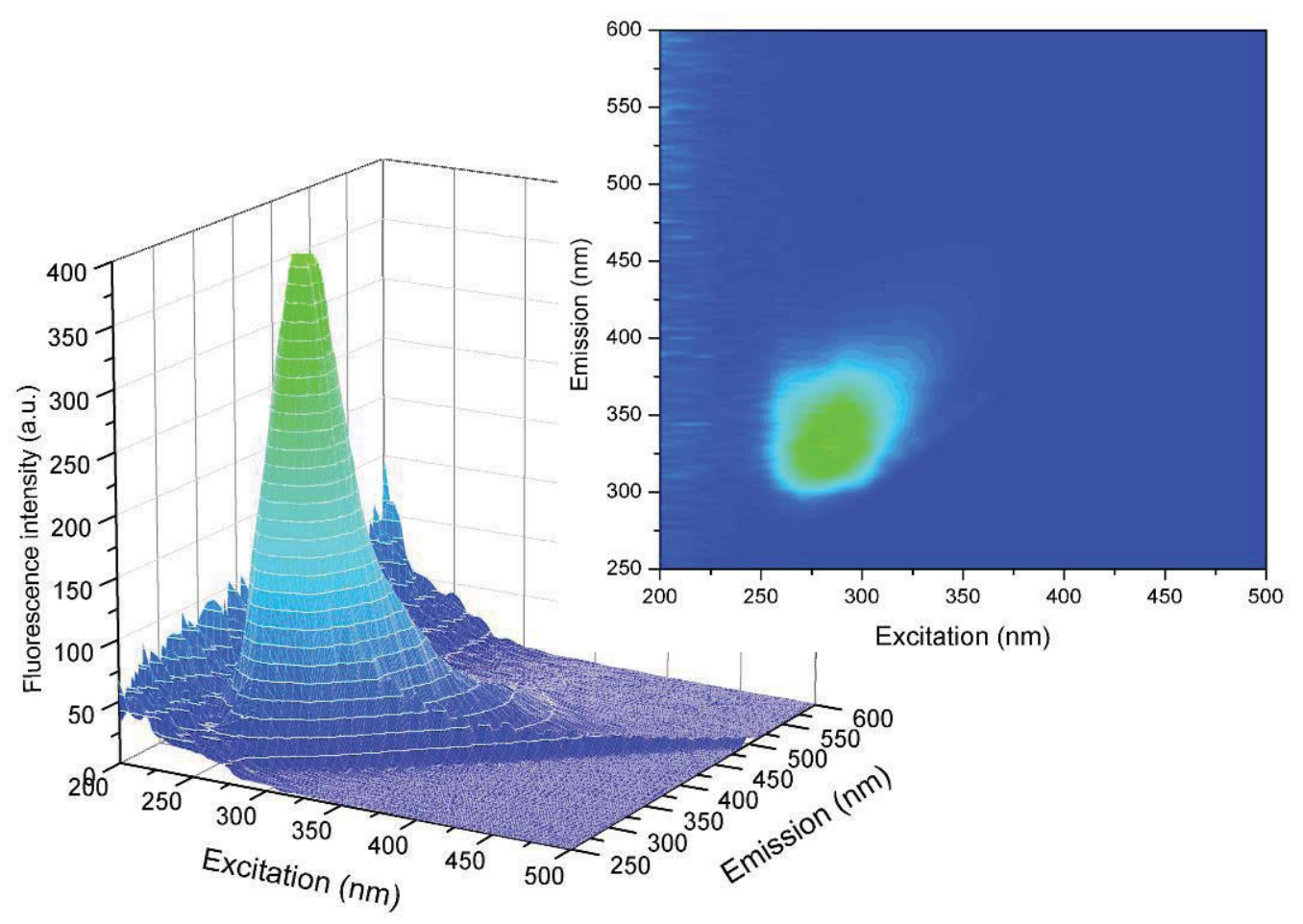

Figure 2: Fluorescence spectrum of non-degraded DEHS [13]

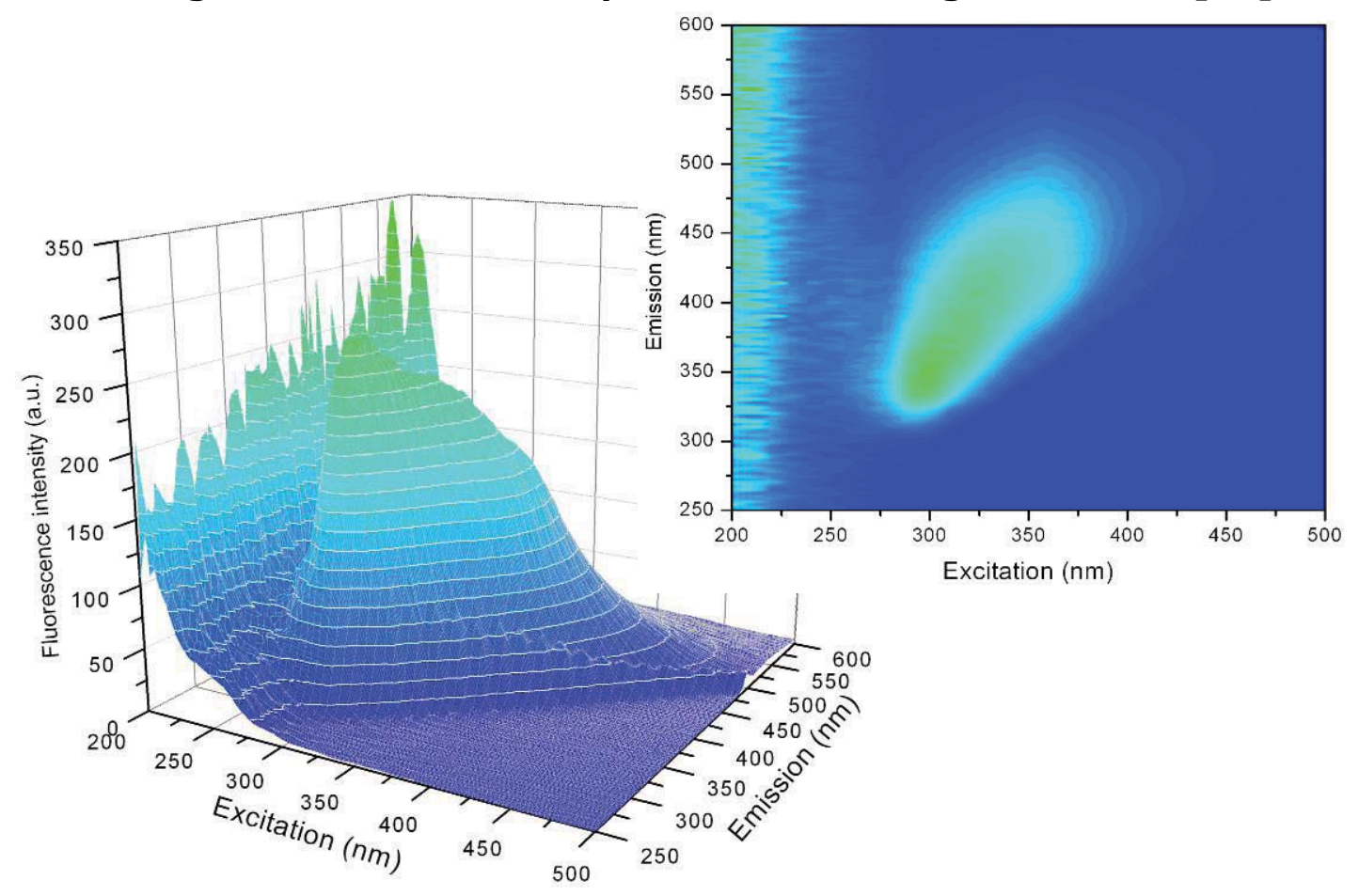

Figure 3: Fluorescence spectrum of degraded DEHS [13] 


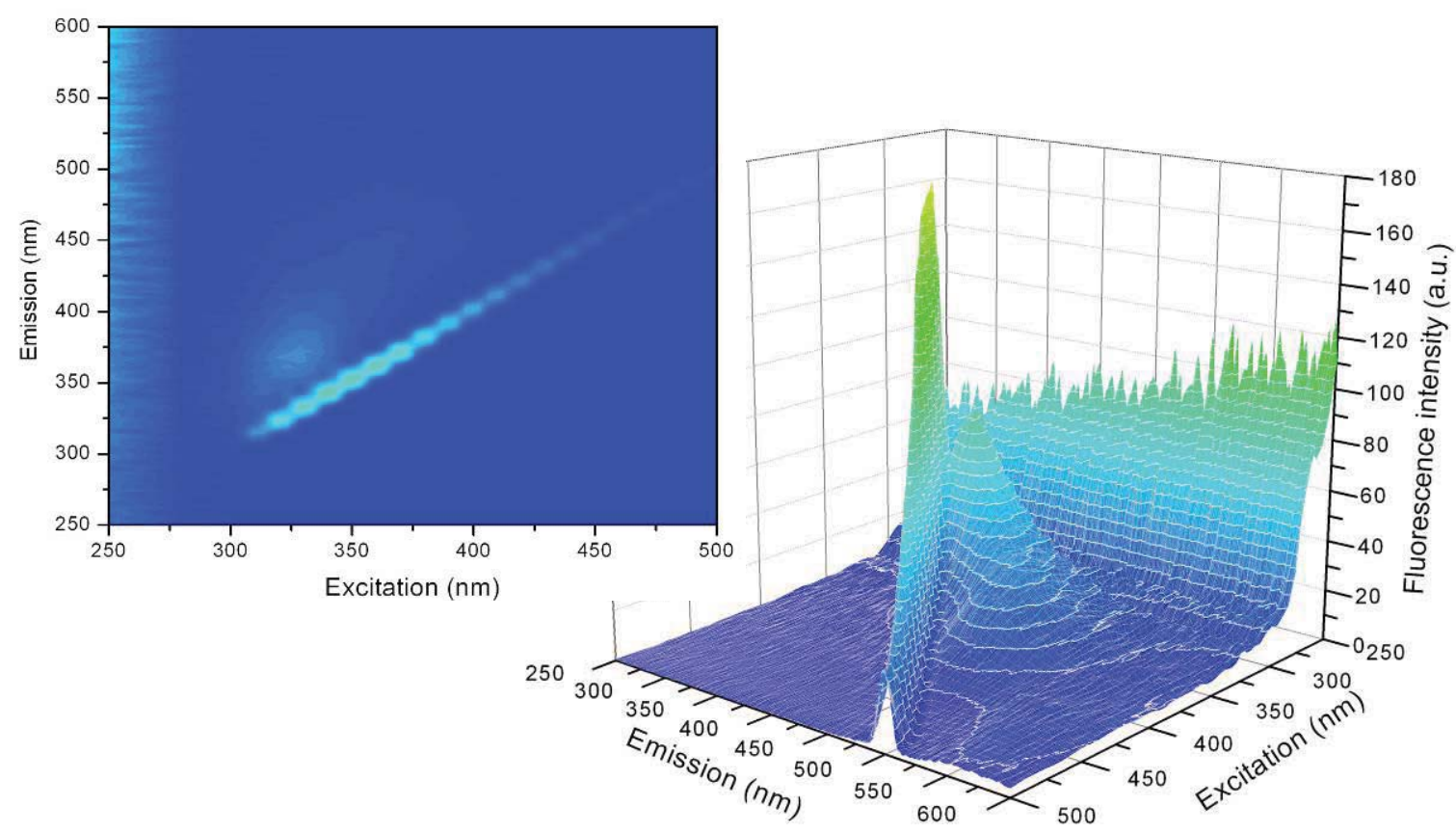

Figure 4: Fluorescence spectrum of DEHS in xylene [13]

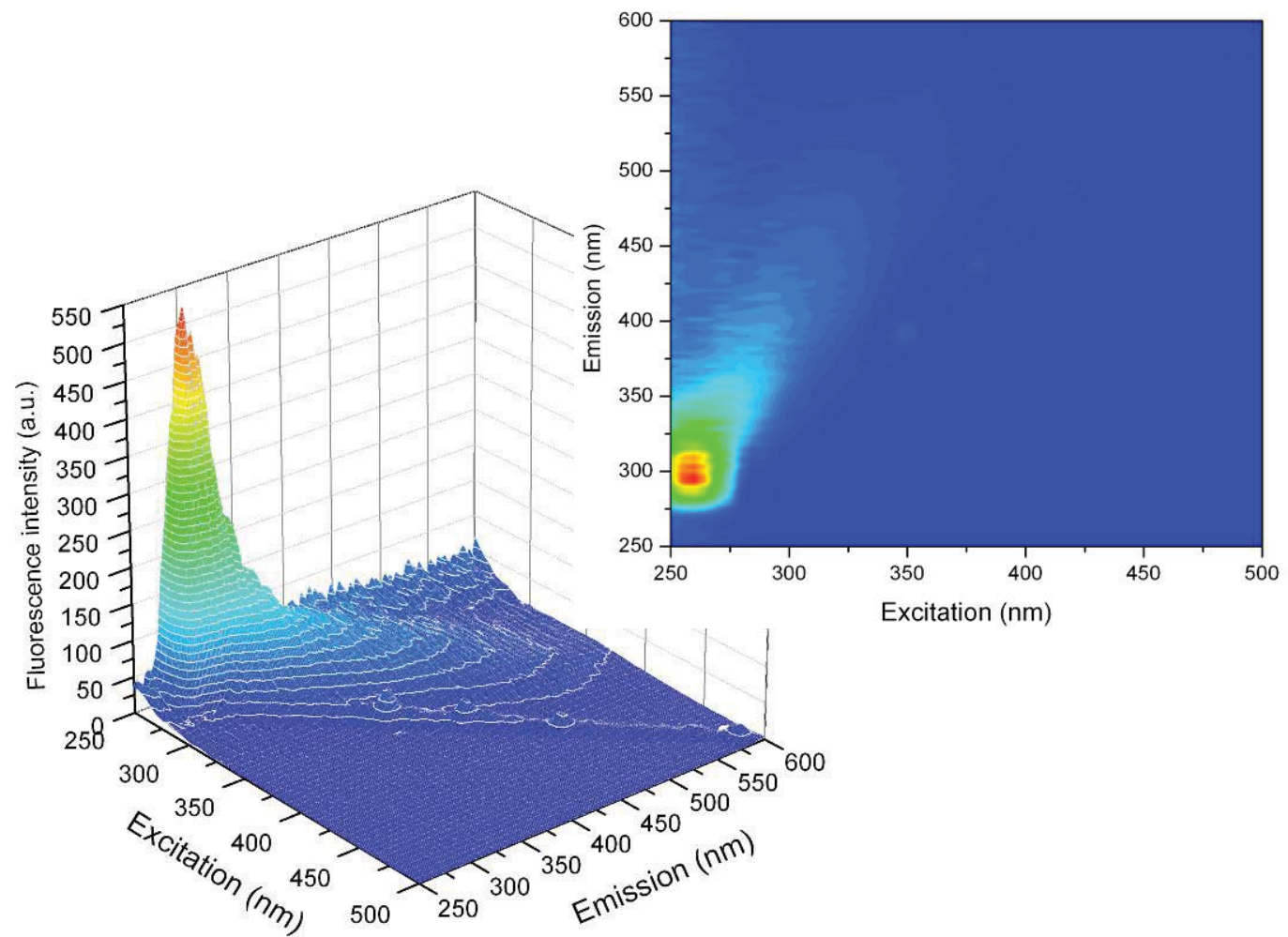

Figure 5: Fluorescence spectrum of DEHS in isopropanol [13] 


\section{Results}

Experiments were performed on a test rig consisting of aerosol generator, aerosol monitor, air-aerosol mixer, segmented model of human lungs [15], ten filters at ten output branches of the model, adjustable flowmeters and a vacuum pump (see Figure 6 ).

Aerosol generator was set to produce $3 \mu \mathrm{m}$ monodisperse particles with concentration $1.10^{5} \mathrm{P} / \mathrm{cm}^{3}$. Flowrates were set individually on each flowmeter to fit the same flow conditions that were used for Phase Doppler Anemometry measurements. Total flowrate through the model was set to 15 LPM (resting conditions) and 30 LPM (deep breath). Experiments were performed in inhalation regime.

The model was dismounted to segments after the experiment and each segment was sonicated in isopropanol to dissolve the deposited DEHS aerosol. Samples created by sonication were analyzed on AMINCO Bowman Series 2

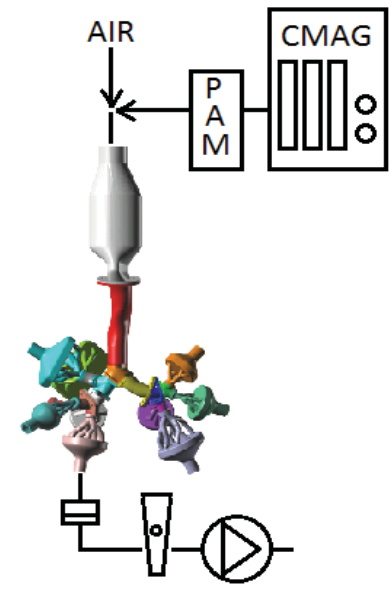
spectrometer and resulting fluorescence intensity for each segment was recorded. We assumed that the fluorescence intensity is directly proportional to the amount of deposited aerosol. The ratio of the segment fluorescence intensity and the sum of intensities of all segments was divided by inner surface area of the segment which equals to aerosol deposition density in the segment. The resulting deposition densities for all segments are presented in Figure 7. However the results did not match published data acquired using different methods (Figure 8).

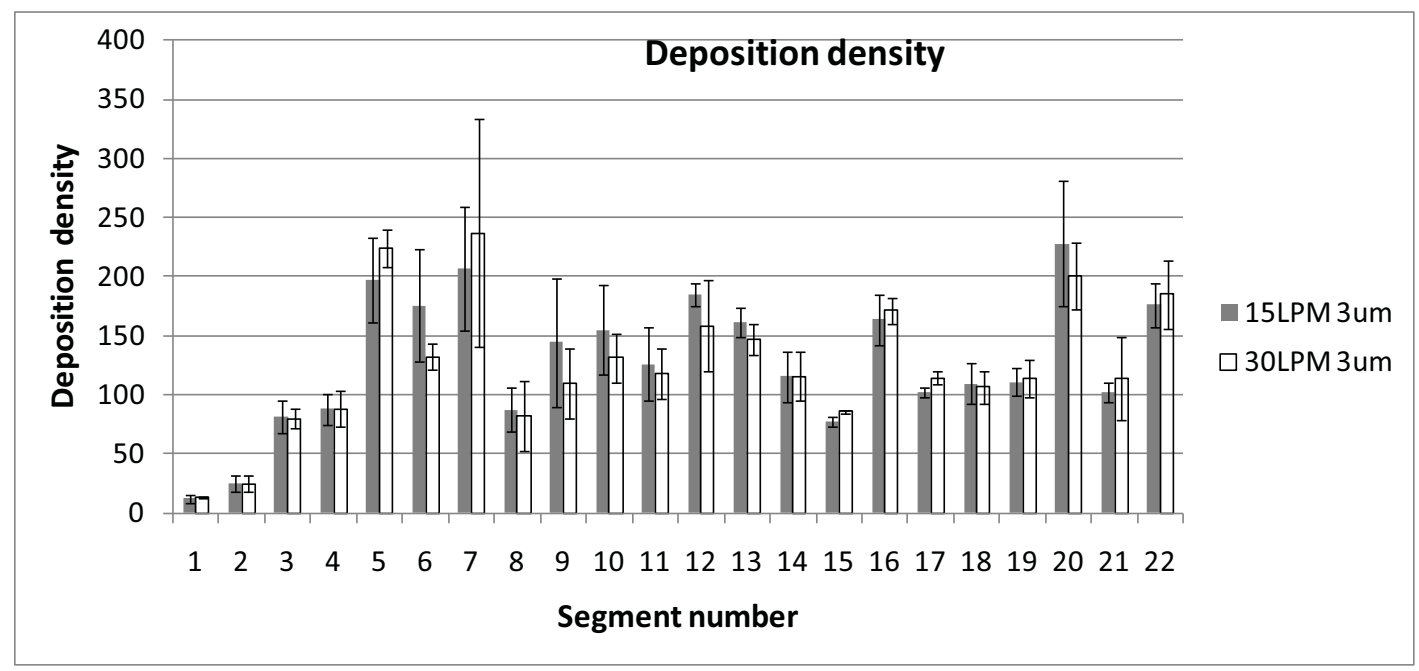

Figure 7: Deposition density results acquired using DEHS fluorescence 


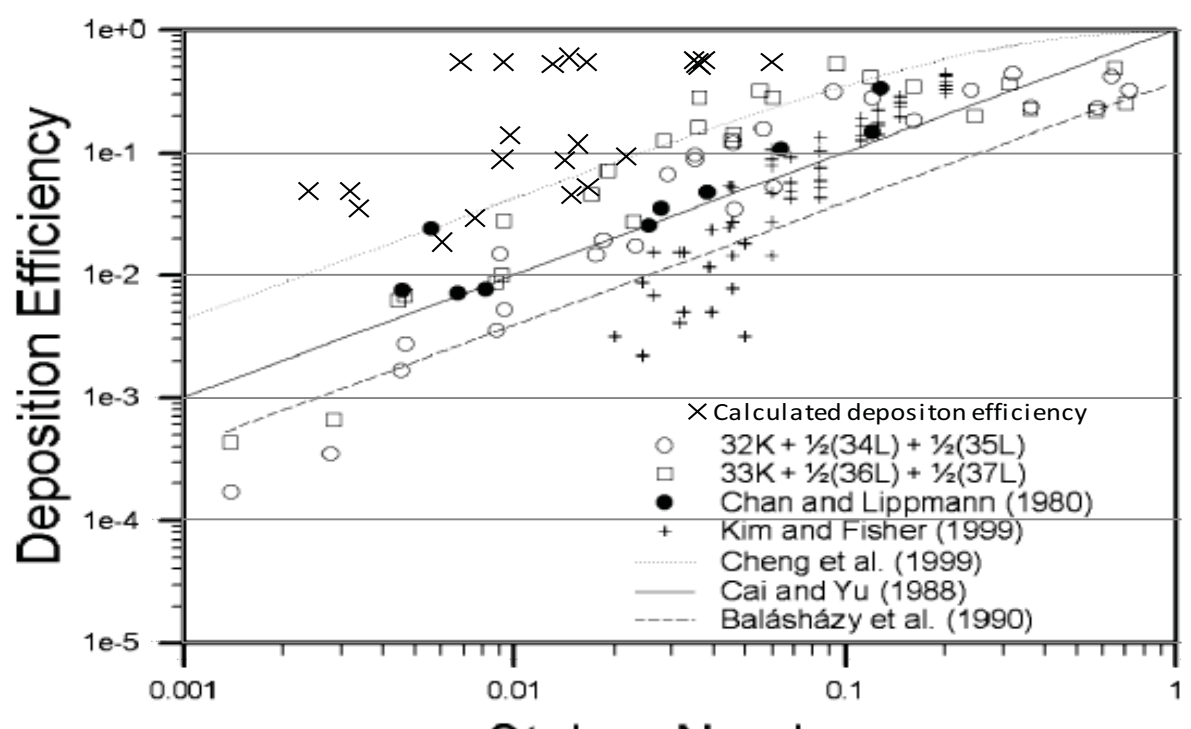

Stokes Number

Figure 8: Comparison with published data (modified from [16])

\section{DISCUSSION}

The reason of the difference in our and published data is probably caused by diverse measuring methods, as the lung models are similar. In our case we did the assumption that the fluorescence intensity is directly proportional to the amount of deposited aerosol, which might be wrong. This was confirmed by present calibration experiments, which demonstrated that there is a very low increase in fluorescence intensity with increasing concentration of DEHS, which leads to inaccurate results in our range of concentrations. Increasing of concentration range is impossible in our case, therefore new suitable fluorescent tracer should be used. Essential characteristic of the new tracer is high resistance to heat, as we need to produce monodisperse particles by condensation in the generator at high temperature.

\section{SUMmARY}

Preliminary experiments confirmed detectability of DEHS by fluorescence spectroscopy. Infrared spectrometry excluded the possibility that the fluorescence is caused by organic impurities in DEHS batch, but the real cause of DEHS fluorescence remains unclear. Isopropanol appears as the most suitable solvent according to 3D fluorescence scan analysis. Aerosol deposition measurement performed using DEHS fluorescence analysis showed considerably different results than expected. The reason is probably very low increase of DEHS fluorescence with increasing concentration. For future measurements of aerosol deposition is therefore necessary to use different fluorescent tracer, however our analysis of DEHS characteristics might be useful in other applications where larger concentration ranges occur. 


\section{ACKNOWLEDGEMENT}

The authors gratefully acknowledge a financial support from the project GA P105/11/1339 funded by the Czech Science Foundation, projects ME 09030 of the program KONTAKT, the project OC10052, COST Action Particles No. MP0806 funded by the Ministry of Education, Youth and Sports of the Czech Republic and the project of the Brno University of Technology FSI-S-11-6. This work was supported by the project "Centre for Materials Research at FCH BUT" No. CZ.1.05/2.1.00/01.0012 from ERDF.

\section{REFERENCES}

[1] Kleinstreuer C., Zhang Z., Li Z., Roberts W.L., Rojas C.: A new methodology for targeting drug-aerosols in the human respiratory system. International Journal of Heat and Mass Transfer, 51, 5578-5589, 2008.

[2] Ally J., Martin B., Behrad Khamesee M., Roa W., Amirfazli A.: Magnetic targeting of aerosol particles for cancer therapy. Journal of Magnetism and Magnetic Materials, 293, 442-449, 2005.

[3] Martin A.R., Finlay W.H.: Enhanced deposition of high aspect ratio aerosols in small airway bifurcations using magnetic field alignment. Journal of Aerosol Science, 39, 679-690, 2008.

[4] Dalby R., Suman J.: Inhalation therapy: technological milestones in asthma treatment. Advanced Drug Delivery Reviews, 55, 779-791, 2003.

[5] Hanania N.A., Ambrosino N., Calverley P., Cazzola M., Donner C.F., Make B.: Treatments for COPD. Respiratory Medicine, 99, S28-S40, 2005.

[6] Hannemann L.A.: What is new in asthma: New dry powder inhalers. Journal of Pediatric Health Care, 13, 159-165, 2004.

[7] Newhouse M.T.: Tennis anyone? The lungs as a new court for systemic therapy. Canadian Medical Association Journal, 161, 1287-1288, 1999.

[8] Jaafar-Maalej C., Andrieu V., Elaissari A., Fessi H.: Assessment methods of inhaled aerosols: technical aspects and applications. Expert Opinion on Drug Delivery, 6, 941-959, 2009.

[9] Jedelsky J., Lizal F., Jicha M.: Power spectral density of velocity fluctuations estimated from phase doppler data. Experimental Fluid Mechanics 2011. Jicin, Czech republic, 2011, In print.

[10] Elcner J., Lizal F., Jedelsky J., Jicha M.: Numerical simulation of air flow in a model of lungs with mouth cavity. Experimental fluid mechanics 2011. Jicin, Czech republic, Technical University of Liberec, 2011, In print.

[11] Bowes S.M., Swift D.L.: Deposition of inhaled particles in the oral airway during oronasal breathing. Aerosol Science and Technology, 11, 157-167, 1989.

[12] Horton K.D., Miller R.D., Mitchell J.P.: Characterization of a condensation-type monodisperse aerosol generator (MAGE). Journal of Aerosol Science, 22, 347-363, 1991.

[13] Lippay J.: Fluorescence spectroscopy in study of aerosol-forming substances. Bachelor's thesis, Brno. Faculty of Chemistry Brno University of Technology, 2011, p. 40.

[14] Stuart B.H., George B., Mcintyre P.: Modern infrared spectroscopy. New York, John Wiley \& Sons, 1996.

[15] Lizal F., Elcner J., Hopke P.K., Jedelsky J., Jicha M.: Development of a realistic human airway model. Proceedings of the Institution of Mechanical Engineers, Part H: Journal of Engineering in Medicine, 2011, In review.

Zhou Y., Cheng Y.S.: Particle deposition in a cast of human tracheobronchial airways. Aerosol Science and Technology, 39, 492-500, 2005. 